\title{
J. M. RASSIAS PRODUCT-SUM STABILITY OF AN EULER-LAGRANGE FUNCTIONAL EQUATION
}

\author{
MATINA J. RASSIAS
}

\begin{abstract}
In 1940 (and 1964) S. M. Ulam proposed the well-known Ulam stability problem. In 1941 D. H. Hyers solved the Hyers-Ulam problem for linear mappings. In 1992 and 2008, J. M. Rassias introduced the Euler-Lagrange quadratic mappings and the JMRassias "product-sum" stability, respectively. In this paper we introduce an Euler-Lagrange type quadratic functional equation and investigate the JMRassias "product-sum" stability of this equation. The stability results have applications in Mathematical Statistics, Stochastic Analysis and Psychology.
\end{abstract}

\section{Introduction AND Preliminaries}

In 1940 (and 1964) Stanislaw M. Ulam [9] proposed the following stability problem, well-known as Ulam stability problem:

"When is true that by slightly changing the hypotheses of a theorem one can still assert that the thesis of the theorem remains true or approximately true?"

In particular he stated the stability question:

"Let $G_{1}$ be a group and $G_{2}$ a metric group with the metric $\rho(.,$.$) . Given a con-$ stant $\delta>0$, does there exist a constant $c>0$ such that if a mapping $f: G_{1} \rightarrow G_{2}$ satisfies $\rho(f(x y), f(x) f(y))<c$ for all $x, y \in G_{1}$, then a unique homomorphism $h: G_{1} \rightarrow G_{2}$ exists with $\rho(f(x), h(x))<\delta$ for all $x \in G_{1}$ ?"

In 1941 D. H. Hyers [3] solved this problem for linear mappings as follows:

Date: Received: 31 December 2009; Revised: 30 March 2010

(c) 2010 N.A.G.

2000 Mathematics Subject Classification. Primary 39B; Secondary 26D..

Key words and phrases. J.M.Rassias "product-sum" stability, Euler-Lagrange type quadratic functional equation. 
Theorem 1.1. (D. H. Hyers, 1941: 3]). If a mapping $f: E \rightarrow E^{\prime}$ satisfies the approximately additive inequality

$$
\|f(x+y)-f(x)-f(y)\| \leq \varepsilon,
$$

for some fixed $\varepsilon>0$ and all $x, y \in E$, where $E$ and $E^{\prime}$ are Banach spaces, then there exists a unique additive mapping $A: E \rightarrow E^{\prime}$, satisfying the formula

$$
A(x)=\lim _{n \rightarrow \infty} 2^{-n} f\left(2^{n} x\right),
$$

and inequality

$$
\|f(x)-A(x)\| \leq \varepsilon
$$

for some fixed $\varepsilon>0$ and all $x \in E$.

No continuity conditions are required for this result.

In 1992, Euler-Lagrange functional equations were introduced ([5], [6]).

Theorem 1.2. (J. M. Rassias, 1992: [5]). Let $X$ be a normed linear space, $Y$ a Banach space, and $f: X \rightarrow Y$. If there exist $\alpha, b: 0 \leq a+b<2$, and $c_{2} \geq 0$ such that

$$
\|f(x+y)+f(x-y)-2[f(x)+f(y)]\| \leq c_{2}\|x\|^{a}\|y\|^{b},
$$

for all $x, y \in X$, then there exists a unique non-linear mapping $N: X \rightarrow Y$ such that

$$
\|f(x)-N(x)\| \leq c\|x\|^{a+b}
$$

and

$$
N(x+y)+N(x-y)=2[N(x)+N(y)]
$$

for all $x, y \in X$, where $c=c_{2} /\left(4-2^{a+b}\right)$.

Note that a mapping $N: X \rightarrow Y$ satisfying (1.6) is called Euler-Lagrange mapping, and a mapping $f: X \rightarrow Y$ satisfying (1.4) is approximately Euler-Lagrange mapping.

In 2008, the JMRassias "product-sum" stability was investigated for the first time $([1],[2],[7],[8])$.

For the theorem that follows, let $(E, \perp)$ denote an orthogonality normed space with norm $\|.\|_{E}$ and $\left(F,\|.\|_{F}\right)$ is a Banach space.

Theorem 1.3. (K. Ravi, M. Arunkumar and J. M. Rassias, 2008: 7]) Let $f$ : $E \rightarrow F$ be a mapping which satisfies the inequality

$$
\begin{aligned}
\| f(m x+y)+f(m x-y)-2 f(x+y)- & 2 f(x-y)-2\left(m^{2}-2\right) f(x)+2 f(y) \|_{F} \\
& \left.\leq \varepsilon\left\{\|x\|_{E}^{p}\|y\|_{E}^{p}+\left(\|x\|_{E}^{2 p}\right)+\|x\| \|_{E}^{2 p}\right)\right\}(1.7
\end{aligned}
$$

for all $x, y \in E$ with $x \perp y$, where $\varepsilon$ and $p$ are constants with $\varepsilon, p>0$ and either $m>1 ; p>1$ or $m<1 ; p>1$ with $m \neq 0 ; m \neq \pm 1 ; m \neq \pm \sqrt{2}$ and $-1 \neq|m|^{p-1}<1$.

Then the limit

$$
Q(x)=\lim _{n \rightarrow \infty} \frac{f\left(m^{n} x\right)}{m^{2 n}}
$$


PRODUCT-SUM STABILITY OF AN EULER-LAGRANGE FUNCTIONAL EQUATION 267 exists for all $x \in E$ and $Q: E \rightarrow F$ is the unique orthogonally Euler-Lagrange quadratic mapping such that

$$
\|f(x)-Q(x)\|_{F} \leq \frac{\varepsilon}{2\left|m^{2}-m^{2 p}\right|}\|x\|_{E}^{2 p}
$$

for all $x \in E$.

Note that the mixed type product-sum function

$$
(x, y) \rightarrow \varepsilon\left[\|x\|_{E}^{p}\|y\|_{E}^{p}+\left(\|x\|_{E}^{2 p}+\|y\|_{E}^{2 p}\right)\right]
$$

was introduced by J. M. Rassias ([1],[2],[7],[8]).

In this paper we introduce an Euler-Lagrange type quadratic functional equation and investigate the JMRassias "product-sum" stability of this equation. The stability results have applications in Mathematical Statistics, Stochastic Analysis and Psychology.

\section{JMRASSIAS PRODUCT-SUM STABILITY OF AN EULER-LAGRANGE TYPE FUNCTIONAL EQUATION}

Let $\mathrm{X}$ be a real normed linear space and $\mathrm{Y}$ a real Banach space.

Definition 2.1. A mapping $f: X \rightarrow Y$ is called approximately Euler-Lagrange type quadratic, if the approximately Euler-Lagrange quadratic functional inequality

$\left\|f(x+y)+\frac{1}{2}[f(x-y)+f(y-x)]-2[f(x)+f(y)]\right\| \leq \varepsilon\left(\|x\|^{\frac{\alpha}{2}}\|y\|^{\frac{\alpha}{2}}+\|x\|^{\alpha}+\|y\|^{\alpha}\right)$

holds for every $x, y \in X$ with $\varepsilon \geq 0$ and $\alpha \neq 2$.

Lemma 2.2. Mapping $Q: X \rightarrow Y$ satisfies the Euler-Lagrange type quadratic equation

$$
Q(x+y)+\frac{1}{2}[Q(x-y)+Q(y-x)]=2[Q(x)+Q(y)]
$$

for all $x, y \in X$ if and only if there exists a mapping $T: X \rightarrow Y$ satisfying the Euler-Lagrange quadratic equation

$$
T(x+y)+T(x-y)=2[T(x)+T(y)]
$$

for all $x, y \in X$ such that $Q(x)=T(x)$ for all $x \in X$.

Proof. $(\Rightarrow)$ Let mapping $Q: X \rightarrow Y$ satisfy the Euler-Lagrange type quadratic equation

$$
Q(x+y)+\frac{1}{2}[Q(x-y)+Q(y-x)]=2[Q(x)+Q(y)]
$$

for all $x, y \in X$. Assume that there exists a mapping $T: X \rightarrow Y$ such that $Q(x)=T(x)$ for all $x \in X$. Observe that for $x=y=0$ and $x=x, y=0$ from (2.2) we obtain respectively

$$
T(0)=Q(0)=0
$$

and

$$
T(-x)=Q(-x)=Q(x)=T(x), \quad \text { for } \quad x \in X .
$$


From $(2.2)$ and $(2.4)$ it is obvious that

$$
\begin{aligned}
T(x+y)+\frac{1}{2}[T(x-y)+T(y-x)] & =2[T(x)+T(y)], \text { or } \\
T(x+y)+\frac{1}{2}[T(x-y)+T(-(x-y))] & =2[T(x)+T(y)], \text { or } \\
T(x+y)+T(x-y) & =2[T(x)+T(y)] .
\end{aligned}
$$

Hence, $T$ satisfies the Euler-Lagrange quadratic equation.

$(\Leftarrow)$ Let mapping $T: X \rightarrow Y$ satisfy the Euler-Lagrange quadratic equation

$$
T(x+y)+T(x-y)=2[T(x)+T(y)]
$$

for all $x, y \in X$. Assume that there exists a mapping $Q: X \rightarrow Y$ such that $Q(x)=T(x)$ for all $x \in X$. Observe that for $x=y=0$ and $x=0, y=x$ from (2.5) we obtain

$$
Q(0)=T(0)=0
$$

and

$$
Q(x)=T(x)=T(-x)=Q(-x), \quad \text { for } \quad x \in X .
$$

Thus, from $(2.5)-(2.7)$ one gets

$$
\begin{aligned}
2[Q(x)+Q(y)] & =2[T(x)+T(y)]=T(x+y)+T(x-y) \\
& =T(x+y)+\frac{1}{2} T(x-y)+\frac{1}{2} T(-(y-x)) \\
& =Q(x+y)+\frac{1}{2}[Q(x-y)+Q(y-x)] .
\end{aligned}
$$

Hence, $Q$ satisfies the Euler-Lagrange type quadratic equation.

Thus the proof of Lemma 2.2 is complete.

Theorem 2.3. Assume that $f: X \rightarrow Y$ is an approximately Euler-Lagrange type additive mapping satisfying (2.1).

Then, there exists a unique Euler-Lagrange type quadratic mapping $Q: X \rightarrow Y$ which satisfies the formula

$$
Q(x)=\lim _{n \rightarrow \infty} f_{n}(x)
$$

where

$$
f_{n}(x)= \begin{cases}2^{-2 n} f\left(2^{n} x\right), & -\infty<\alpha<2 \\ 2^{2 n} f\left(2^{-n} x\right), & \alpha>2\end{cases}
$$

for all $x \in X$ and $n \in N=\{0,1,2, \ldots\}$, which is the set of natural numbers and

$$
\|f(x)-Q(x)\| \leq \frac{3 \varepsilon}{\left|2^{\alpha}-4\right|}\|x\|^{\alpha}
$$

for some fixed $\varepsilon>0, \alpha \neq 2$ and all $x \in X$.

$Q: X \rightarrow Y$ is a unique Euler-Lagrange type quadratic mapping satisfying equation

$$
Q(x+y)+\frac{1}{2}[Q(x-y)+Q(y-x)]=2[Q(x)+Q(y)] .
$$


Proof. We start our proof considering: $-\infty<\alpha<2$.

Step 1. By substituting $x=y$ in (2.1), we can observe that

$$
\|f(2 x)+f(0)-4 f(x)\| \leq 3 \varepsilon\|x\|^{\alpha},
$$

from which for $x=0$ it occurs that

$$
f(0)=0
$$

and in extension

$$
\left\|f(x)-2^{-2} f(2 x)\right\| \leq \frac{3}{4} \varepsilon\|x\|^{\alpha} .
$$

Hence, for $n \in N-\{0\}$

$$
\begin{aligned}
\left\|f(x)-2^{-2 n} f\left(2^{n} x\right)\right\| & \leq\left\|f(x)-2^{-2} f(2 x)\right\|+\left\|2^{-2} f(2 x)-2^{-4} f\left(2^{2} x\right)\right\|+\ldots \\
& +\left\|2^{-2(n-1)} f\left(2^{n-1} x\right)-2^{-2 n} f\left(2^{n} x\right)\right\| \\
& \leq \frac{3}{4}\left(1+2^{\alpha-2}+\ldots+2^{(n-1)(\alpha-2)}\right) \varepsilon\|x\|^{\alpha} \\
& =\frac{3}{4-2^{\alpha}}\left(1-2^{n(\alpha-2)}\right) \varepsilon\|x\|^{\alpha} .
\end{aligned}
$$

Thus,

$$
\left\|f(x)-2^{-2 n} f\left(2^{n} x\right)\right\| \leq \frac{3}{4-2^{\alpha}}\left(1-2^{n(\alpha-2)}\right) \varepsilon\|x\|^{\alpha},
$$

for $n \in N-\{0\}$ and $-\infty<\alpha<2$.

Step 2. Following, we need to show that if there is a sequence $\left\{f_{n}\right\}: f_{n}(x)=$ $2^{-2 n} f\left(2^{n} x\right)$, then $\left\{f_{n}\right\}$ converges.

For every $n>m>0$, we can obtain

$$
\begin{aligned}
\left\|f_{n}(x)-f_{m}(x)\right\| & =\left\|2^{-2 n} f\left(2^{n} x\right)-2^{-2 m} f\left(2^{m} x\right)\right\| \\
& =2^{-2 m}\left\|f\left(2^{m} x\right)-2^{-2(n-m)} f\left(2^{(n-m)} 2^{m} x\right)\right\| \\
& \leq 2^{m(\alpha-2)} \frac{3 \varepsilon}{4-2^{\alpha}}\left(1-2^{(n-m)(\alpha-2)}\right)\|x\|^{\alpha} \\
& <2^{m(\alpha-2)} \frac{3 \varepsilon}{4-2^{\alpha}}\|x\|^{\alpha} \rightarrow 0,
\end{aligned}
$$

for $m \rightarrow \infty$, as $\alpha<2$. Therefore, $\left\{f_{n}\right\}$ is a Cauchy sequence. Since $Y$ is complete we can conclude that $\left\{f_{n}\right\}$ is convergent. Thus, there is a well-defined $Q: X \rightarrow Y$ such that $Q(x)=\lim _{n \rightarrow \infty} 2^{-2 n} f\left(2^{n} x\right)$, for $\alpha<2$.

Step 3. Observe that

$$
\left\|f(x)-f_{n}(x)\right\|=\left\|f(x)-2^{-2 n} f\left(2^{n} x\right)\right\| \leq \frac{3 \varepsilon}{4-2^{\alpha}}\left(1-2^{n(\alpha-2)}\right)\|x\|^{\alpha},
$$

from which by letting $n \rightarrow \infty$ we obtain

$$
\|f(x)-Q(x)\| \leq \frac{3 \varepsilon}{4-2^{\alpha}}\|x\|^{\alpha} .
$$


Step 4. Claim that mapping $Q: X \rightarrow Y$ satisfies (2.10). In fact, by letting $x \rightarrow 2^{n} x$ and $y \rightarrow 2^{n} y$, from (2.1), we have:

$$
\begin{array}{r}
\left\|f\left(2^{n}(x+y)\right)+\frac{1}{2}\left[f\left(2^{n}(x-y)\right)+f\left(2^{n}(y-x)\right)\right]-2\left[f\left(2^{n} x\right)+f\left(2^{n} y\right)\right]\right\| \\
\leq \varepsilon\left(\left\|2^{n} x\right\|^{\frac{\alpha}{2}}\left\|2^{n} y\right\|^{\frac{\alpha}{2}}+\left\|2^{n} x\right\|^{\alpha}+\left\|2^{n} y\right\|^{\alpha}\right) .
\end{array}
$$

Next, by multiplying with $2^{-2 n}$ we obtain

$$
\begin{aligned}
0 \leq \| 2^{-2 n} f\left(2^{n}(x+y)\right) & +\frac{1}{2}\left[2^{-2 n} f\left(2^{n}(x-y)\right)+2^{-2 n} f\left(2^{n}(y-x)\right)\right] \\
& -2\left[2^{-2 n} f\left(2^{n} x\right)+2^{-2 n} f\left(2^{n} y\right)\right] \| \\
& \leq 2^{n(\alpha-2)} \varepsilon\left(\|x\|^{\frac{\alpha}{2}}\|y\|^{\frac{\alpha}{2}}+\|x\|^{\alpha}+\|y\|^{\alpha}\right)
\end{aligned}
$$

and by letting $n \rightarrow \infty$, for $\alpha<2$ we can conclude that an $Q: X \rightarrow Y$ truly exists such that: $Q(x)=\lim _{n \rightarrow \infty} 2^{-2 n} f\left(2^{n} x\right)$ satisfies the Euler-Lagrange type quadratic property

$$
Q(x+y)+\frac{1}{2}[Q(x-y)+Q(y-x)]=2[Q(x)+Q(y)] .
$$

Therefore, existence of Theorem holds.

Step 5. We need to prove that Q is unique.

Observe, from (2.15), that for $a) x=y=0, b) x=x, y=0$ and $c$ ) $x=y$, we obtain:

$$
\text { a) } Q(0)=0, \quad \text { b) } Q(-x)=Q(x) \text { and } \quad \text { c) } Q(2 x)=2^{2} Q(x) \text {, }
$$

respectively. Therefore, by induction, by claiming that $Q\left(2^{n-1} x\right)=2^{2(n-1)} Q(x)$, we can show that

or equivalently

$$
Q\left(2^{n} x\right)=2^{2} Q\left(2^{n-1} x\right)=2^{2 n} Q(x)
$$

$$
Q(x)=2^{-2 n} Q\left(2^{n} x\right) .
$$

Assume, now, the existence of another $Q^{\prime}: X \rightarrow Y$, such that $Q^{\prime}(x)=2^{-2 n} Q^{\prime}\left(2^{n} x\right)$. With the aid of the (2.14)-(2.16) and the triangular inequality, one gets

$$
\begin{aligned}
0 \leq\left\|Q(x)-Q^{\prime}(x)\right\| & =\left\|2^{-2 n} Q\left(2^{n} x\right)-2^{-2 n} Q^{\prime}\left(2^{n} x\right)\right\| \\
& \leq\left\|2^{-2 n} Q\left(2^{n} x\right)-2^{-2 n} f\left(2^{n} x\right)\right\|+\left\|2^{-2 n} f\left(2^{n} x\right)-2^{-2 n} Q^{\prime}\left(2^{n} x\right)\right\| \\
& \leq 2^{n(\alpha-2)} \frac{6 \varepsilon}{4-2^{\alpha}}\|x\|^{\alpha} \\
& \rightarrow 0,
\end{aligned}
$$

as $n \rightarrow \infty,(\alpha<2)$. Thus, the uniqueness of $Q$ is proved and the stability of Euler-Lagrange type quadratic mapping $Q: X \rightarrow Y$ is established.

The proof for the case of $\alpha>2$ is similar to the proof for $-\infty<\alpha<2$.

In fact, we can find the general inequality

$$
\left\|f(x)-2^{2 n} f\left(2^{-n} x\right)\right\| \leq \frac{3 \varepsilon}{2^{\alpha}-4}\left(1-2^{n(2-\alpha)}\right)\|x\|^{\alpha},
$$

for all $n \in N-\{0\}$. Thus from this inequality (2.17) and the formula

$$
Q(x)=\lim _{n \rightarrow \infty} 2^{2 n} f\left(2^{-n} x\right),
$$


for $n \rightarrow \infty$, we get the inequality

$$
\|f(x)-Q(x)\| \leq \frac{3 \varepsilon}{2^{\alpha}-4}\|x\|^{\alpha}, \quad \text { for } \quad \alpha>2 .
$$

The rest of the proof for $\alpha>2$ is omitted as similar to the above mentioned proof for $-\infty<\alpha<2$.

\section{REFERENCES}

[1] M. Arunkumar, Hyers-Ulam-Rassias (HUR), Ulam-Gavruta-Rassias (UGR) and JMRassias (JMR) Stabilities of Various Functional Equations, PhD Thesis, Thiruvalluvar University, Vellore - 632 004, 2009.

[2] M. E. Gordji, S. Zolfaghari, J.M. Rassias, and M. B. Savadkouhi, (2009). Solution and Stability of a Mixed Type Cubic and Quartic functional equation in Quasi-Banach spaces, Abstract and Applied Analysis, 2009, Art. ID 417473, 1-14, Doi: 10.1155/2009/417473.

[3] D. H. Hyers, On the stability of the linear functional equations, Proc. Nat. Acad. Sci., 27, 222-224: The Stability of Homomorphisms and Related Topics, 'Global Analysis - Analysis of Manifolds', Teubner-Texte zur Mathematik, 57 (1983), 140-153.

[4] J. M. Rassias, Solution of a problem of Ulam, J. Approx. Theory, 57 (1989), 268-273.

[5] J. M. Rassias, On the stability of the Euler-Lagrange functional equation, Chinese J. Math., 20 (1992), 185-190.

[6] M.J. Rassias, and J.M. Rassias, On the Ulam stability for Euler-Lagrange type quadratic functional equations, Austral. J. Math. Anal. Appl., 2 (2005), 1-10.

[7] K. Ravi, M. Arunkumar, M. and J.M. Rassias, . Ulam stability for the orthogonally general Euler-Lagrange type functional equation, Intern. J. Math. Stat., 3 (2008), 36-46.

[8] M. B. Savadkouhi, M. E. Gordji, J. M. Rassias, and N. Ghobadipour, Approximate ternary Jordan derivations on Banach ternary algebras, J. Math. Phys., 50, (2009), 1-9.

[9] S. M. Ulam, A Collection of Mathematical problems, Interscience Publisher, Inc., No. 8, New York; Problems in Modern Mathematics, Wiley and Sons, New York, 1964.

Department of Statistics, University of Glasgow, Mathematics Building, OfFice No. 208, University Gardens, Glasgow G12 8QW, U.K.

E-mail address: s.rassias@stats.gla.ac.uk ; rassias.matina@gmail.com 